\title{
A DSS based on Entropy Method in EIS in Chinese Financial Sectors
}

\author{
Renqian Zhang ${ }^{1}$ and Hongxun Jiang ${ }^{2}$ \\ 1 School of Economics \& Management, BeiHang University, Beijing \\ 100083, China zhangrenqian@buaa.edu.cn \\ WWW home page: http://rsc.buaa.edu.cn/rscfront/find aspx \\ 2 Information School, Renmin University of China, Beijing 1000872, China \\ jianghx@ruc.edu.cn \\ WWW home page: http://info.ruc.edu.cn/teachers/jianghx.asp
}

\begin{abstract}
At the time of commercial banks verifying and assessing the loan applications of enterprises, there is one important problem faced that is how to choose the qualified ones from the numerous applications, which could be named of the Decision-making Program of Loan-granting (DPL). The members of evaluating and granting loan group make up from different departments of banks and different specialized experts. How to synthesize suggestion of expert, draw and reflect expert panel's result of suggestion most, it has been a focus question of the decision theory all the time. The problems of loan's group decision were briefly introduced, and then the authors proposed an entropy-based DSS for examining and approving loan applications. The core mathematics models were emphasized which applied a combination of the multi-attribute group decision-making plural entropy model and traditional group eigenvalue method for the loan evaluation purpose. And then its function, structure modules, key technology and solution method were reviewed in detail. Finally, an example is proposed to show how the entropy method can be applied to the evaluation practice.
\end{abstract}

\section{Introduction}

Credit risk means refer to a kind of default possibility of debtors or borrowers fail to debt or banks' loan in full amount in time with various kinds of reason. At once a contract broken, creditor or bank will bear loss of financial affairs because of failing to get the income expected.

Facing contingent credit risk, foreign commercial banks generally adopt an expert-assess-method by giving credit mark at the time of examining and approving

Please use the following format when citing this chapter: 
a loan application, abbreviate named of expert system model. It is an effectual credit risk analysis and management system formed among commercial banks' long-term credit activity. The greatest characteristic of this method is that, credit officers with abundant experience who trained for a long time control policy-making power of banks' credit. Therefore, in the course of credit decision, credit officer's professional knowledge and subjective judgments, other key elements and their weight are the most important decisive factors. Under the expert system, because of every commercial bank's own terms different, the content of credit analysis carrying on is also different. Although the content varies, most banks' credit analysis focuses on " 5 C" of loan applicants, namely Character, Capacity, Capital or Cash, Collateral, Condition.

In the essence, the credit method adopted currently by domestic commercial banks is also a kind of the 5C-based expert system models, which changes only in form, has distinct Chinese characteristics further. In China, an internal special committee holds the power of making credit policy. At first, the loan secretariat collect and verify application materials which are enterprise financial statements consist of balance sheet, profit-and-loss statement, cash flow statement, etc. After compiled complete, these materials give to the committee for credit examination and approval for vote meeting. Finally, the committee members decide whether approve the application or how much is awarded for comprehensive accrediting amount.

At the time of commercial bank verifying and assessing the loan applications of enterprises, there is one important problem faced that is how to choose the qualified ones from the numerous applications, which could be named of the Decision-making Program of Loan-granting (DPL). But these committee members come from different departments of banks, and are different specialized experts. How to synthesize suggestion of expert, draw and reflect expert panel's result of suggestion most, it has been a focus question of the decision theory all the time. The author proposed an entropy-based DSS of examining and approving loan applications, which are suitable for distinct Chinese characteristics, named of LEDAS (Loan Examination Decision Analysis System). This system contribute $s$ to change traditional loan application for risk assesses and controls, improve banks' credit risk management level and working efficiency. This paper first proposed a logic and software structure of LEDAS. Then its function, structure modules, key technology and solution method are analyzed and designed in detail. At last, a case study is proposed to show how the DSS can be applied to evaluation practice. The results of the study show that the suggested system can improve the decision quality. Moreover, the author probes into some questions existing at present and further research directions.

\section{Systematic modeling}

In application materials, various kinds of financial statements of applicant are the most important factors for approval, and are also the primary standard whether 
granting the loan while evaluating. In addition, credit officers will consider other factors, for instance: loan motive, reputation of debtor, experience of similar project, the time finished, refund plan etc. It is the process of credit loan examination and approval. Fig. 1 describes a general course of "LEDAS of Industrial and Commercial Bank of China" in detail.

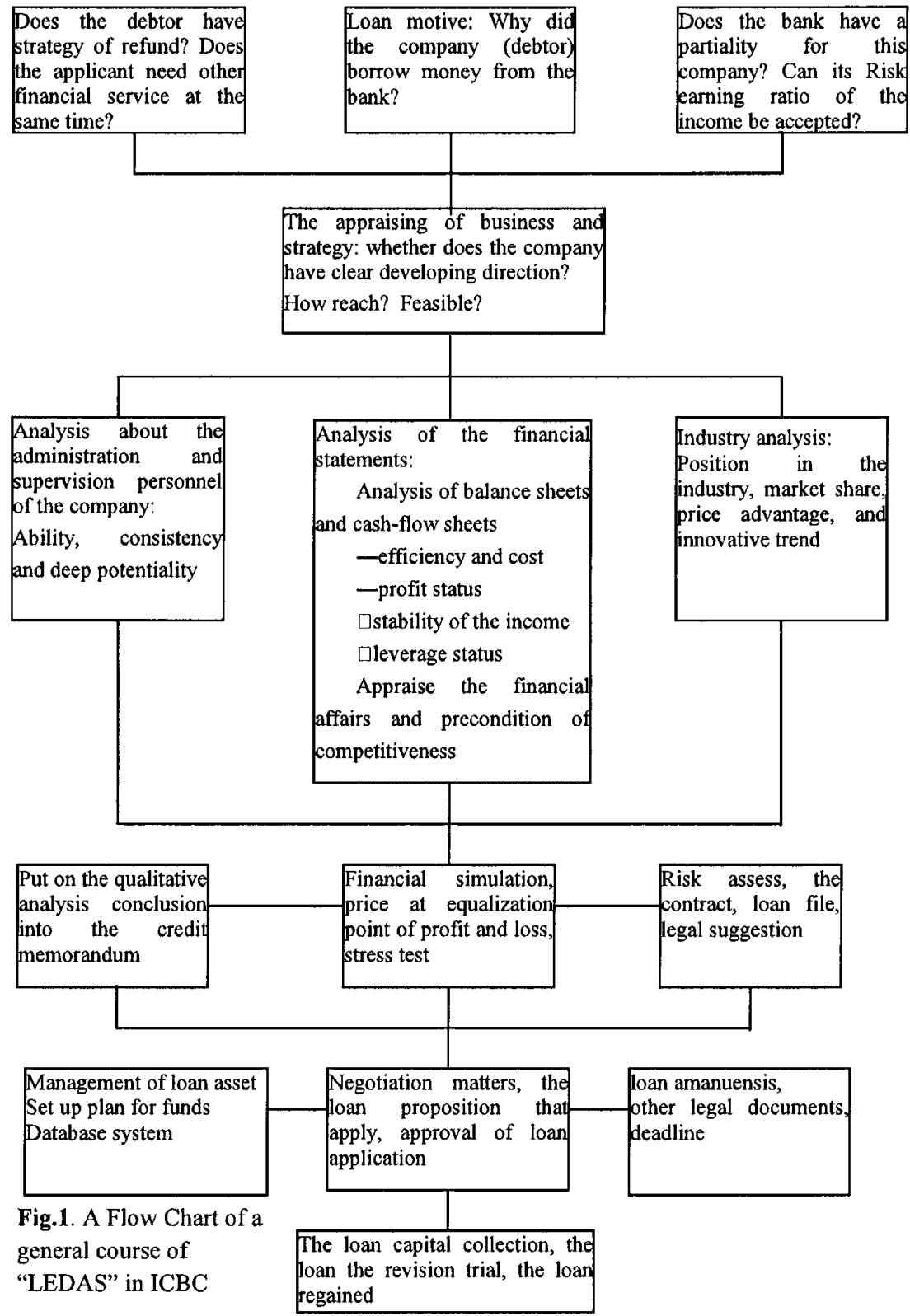


Because these members of the for credit examination and approval committee are all experts coming from different fields, and their ability, level and partiality are all inconsistent, the most essential and difficult problem of LEDAS is how to synthesize suggestion of expert, draw and reflect expert panel's result of suggestion most. This paper proposed a new method for the process of Loan Examination Decision Analysis, which combines group eigenvalue method with multi-attribute group decision-making plural entropy method.

The decision-making system $G$ composing by $m$ experts of $S_{1}, S_{2}, \cdots S_{m}$. evaluate n subjects $B_{1}, B_{2}, \ldots, B_{n}$. No. I experts $S_{J}$ evaluate subjects $B_{j}$ as, $x_{i j} \in[I, J](i=1,2, \ldots, m$; $j=1,2, \ldots, n)$. The larger value is the more excellent aim $B_{j . .} S_{i}$ and grade of its group $G$ are made of $\mathbf{n}$ lines vector $x i$ and $\mathbf{m} \times \mathbf{n}$ matrix $X$ :

$$
\begin{gathered}
x_{i}=\left(x_{i 1}, x_{i 2}, \ldots, x_{i n}\right)^{T} \\
X=\left(x_{i j}\right)_{m \times n}=\left(\begin{array}{cccc}
x_{11} & x_{12} & \cdots & x_{1 n} \\
x_{21} & x_{22} & \cdots & x_{2 n} \\
\vdots & \vdots & \vdots & \vdots \\
x_{m 1} & x_{m 2} & \cdots & x_{m n}
\end{array}\right)
\end{gathered}
$$

They are the conclusion giving by the experts and groups in the decision-making processing. They represent each individual gauge to the evaluated matter.

Because of the limitations of each professional level, business scope, experience, range of knowledge, integration capability, and some other factors closely related with making policy such as state of mind, mood and partiality, in actual life the expert whose dependability is up to 1 of decision does not exist. However, we could image there is a definitely ideal one, whose decision is most exact and right. His dependability is up to 1 . The best decision-making expert called ideal expert $S_{*}$, his grade vector is $x_{*}$ :

$x_{*}=\left(x_{*_{1}}, x_{*_{2}}, \ldots, x_{*_{n}}\right)^{T}$

Known by reference [1], the ideal expert's grade vector $x_{*}$ is got through calculating formula (1).

$$
\underset{\|b\|_{2}=1}{\operatorname{Max}} \sum_{i=1}^{m}\left(b^{T} x_{i}\right)^{2}=\sum_{i=1}^{m}\left(x_{*}^{T} x_{i}\right)^{2}=\rho_{\max }
$$

In formula (1), $\rho_{\max }$ is the greatest eigenvalue of the matrix $x^{T} x ; x_{*}$ is the positive eigenvector of the $\rho_{\max }$. And

$$
\|x *\|_{2}=1
$$

Reference [2] defines uncertainty of decision conclusion of the expert $S_{i}$ as his decision entropy $H_{i}$. For the sake of proposing the calculation formula of $H_{i}$, 
underneath we import some symbols from reference [2]. First we transfer $x_{i}$ into identity matrix, namely

$D_{i}=\left(d_{i 1}, d_{i 2}, \cdots, d_{i n}\right)^{T} \in E^{n}$

$D=\left(D_{1}, D_{2}, \cdots, D_{m}\right)^{T}=\left(d_{i j}\right)_{m \times n}$

In the formula, ${ }^{i}=*, 1,2, \cdots, m, d_{i j}=x_{i j} / \sqrt{x_{i 1}^{2}+x_{i 2}^{2}+\cdots+x_{i n}^{2}}, j=1,2, \cdots, n$

So $0 \leq d_{i j} \leq 1$, and

$$
D_{i}^{T} D_{i}=\left\|D_{i}\right\|_{2}^{2}=\sum_{j=1}^{n} d_{i j}^{2}=1 \quad i=*, 1,2, \cdots, m
$$

Then, according to the grade point of the expert $S_{i}(i=*, 1,2, \cdots, m)$, $N_{i}=\left(N_{i 1}, N_{i 2}, \cdots, N_{i n}\right)$ represents the good and bad places of appraised objects $B_{1}, B_{2}, \cdots, B_{n}$. The object, which the expert $S_{i}$ offers the tallest grade point, is recorded as $B_{j}$

$x_{i j^{*}}=\max _{1 \leq j \leq n} x_{i j} \quad i=*, 1,2, \cdots, m$

So $N_{i j^{*}}=1$. On the contrary, for the object $B_{j 0}$ which is attained the lowest grade point, $N_{i j 0}=n$.

Definition 1: The decision level vector of the expert $S_{i}$ is

$E_{i}=\left(e_{i 1}, e_{i 2}, \cdots, e_{i n}\right)$

$e_{i j}=1-\left|N_{*_{j}}-N_{i j}\right|-\left|d_{*_{j}}-d_{i j}\right|$

In the formula, $i=1,2, \cdots, m ; j=1,2, \cdots, n$

From definition 1 we can conclude, in the decision level vector $E_{*}=(1,1, \cdots, 1)$ of the ideal expert $S_{*}$, each vectorial weight value is up to maximum 1, his decision result is the best one. More low is individual decision level of the expert $S_{i}$, more big is the gap with the ideal expert $S_{*}$, and more little is each vectorial weight value of the decision level vector $E_{i}$. Therefore, the decision level vector $E_{i}$ is objective to completely reflect the conclusion's level of the expert $S_{i}$ to the appraised objects $B_{1}, B_{2}, \cdots, B_{n}$.

Definition 2: The decision level of the expert $S_{i}$, can be measured by the uncertainty of his conclusion that is decision-entropy $H_{i} . H_{i}$ is equal to the sum of broad sense entropy of each vectorial weight value of the decision level vector $E_{i}$.

$$
H_{i}=\sum_{j=1}^{n} h_{i j} \quad i={ }^{*}, 1,2, \cdots, m
$$


In the formula,

$h_{i j}=\left\{\begin{array}{l}-e_{i j} \ln e_{i j}, \text { 当 } 1 / e \leq e_{i j} \leq 1 \text { 时 } \\ \frac{2}{e}-e_{i j}\left|\ln e_{i j}\right|, \text { 当 } e_{i j}<1 / e \text { 时 }\end{array}\right.$

According to Reference [2], when the decision uncertainty of the expert $S_{i}$ is lower, the reliability is higher, and the decision-entropy $H_{i}$ is smaller. Each expert $S_{i}$ 's decision- entropy $H_{i}$ can be calculated, and it reflects his decision level.

Combining the multi-attribute group decision-making plural entropy model with traditional group eigenvalue method applying for the loan evaluation purpose, there are two applied methods according to the different types of the evaluation course.

1) If the experts synthesize in many aspects attribute of the applicants, they give a mark by the overall performance of each one. Then, the mark vector $x_{*}$ can be calculated through GEM. At last, the corresponding applicant of the biggest vectorial weight value could be approved.

2) If the experts give a mark one by one for various fields attribute in applicants, the weight $\omega_{i}$ of each expert's opinion in various fields attribute can be calculated:

$$
\omega_{i j}=c_{i j}, c_{i j}=1 / h_{i j}, i=1,2, \cdots, m ; j=1,2, \cdots, n
$$

In formula (3), $h_{i j}$ is the attribute $j$ 's entropy value of the expert $S_{i} ; \omega_{i j}$ is the attribute $j$ 's weight of the expert $S_{i}$.

So the expert panel gives a mark value for the applicant $a$ as

$$
E_{a}=\sum_{j} \sum_{i} x_{i j}^{a} \omega_{i j}
$$

In formula (4), $x_{i j}$ is the expert $S_{i}$ 's mark value for the attribute $j$ of the applicant $a$; $\omega_{i j}$ is the weight of the expert $S_{i}$ when evaluating the attribute $j$.

\section{System frame structure Design of LEADS}

The goal of LEADS is to classify the customer groups in some area, seek top group of customers, measure the risk of credit products, and acquire optimal financial structure of credit products. According to former relevant indexes of loan customer and potential loan customer as criterion, the system go on and consider every possible angle in whole bank all project of support, subsidiary credit policymaker carry on loan examinational and approval decision. The LEADS can be divided into several parts (see Fig. 2): 1) Human and computer mutual system. It is mutual interface between system and end users. The system commands the user to input the necessary data (using on computer) and information (using on control), and show users to operation situation and final outcome. 2) The management subsystem of 
applicant enterprise's information, including enterprise's basic information, historical credit materials, operation situation, enterprise group operation information, legal representative of enterprise, enterprise surtax materials, etc. 3) The information subsystem of loan projects, including loan application form, the report of credit application, information of guarantee enterprise, loan guarantee situation, the followup tracing circumstance, etc. 4) The decision support subsystem of loan projects' examination and approval, including synthetically analyses module, regional index data, comprehensive inquiry system, office automated system. 5) The approval subsystem of loan projects makes up by two parts: the voting of credit approval and the management of the voting results. 6) System maintaining subsystem, including dictionary management, authority management, roles of users, control information of the report forms.

\section{An Example Study}

Using above giving criterion system, the case is about to appraise and analyze three enterprises' applications of loan projects nowadays. In order to raise the veracity, our subject group has done actual survey and randomly selected a lot of criterion data of three enterprises and the concrete criterion data of their loan projects situation in four years. The credit examination and approval committee is made up by six experts. The experts evaluate them respectively from two respects including debtor's financial situation and development prospect of the loan project, and then give a mark (maximum 5, minimum 1). The criterion mark values based the opinion of experts have been listed in Table 1 .

Table 1. The experts' grade table for debtors and the loan projects

\begin{tabular}{lccccccc}
\hline $\begin{array}{l}\text { Economic } \\
\text { indicators }\end{array}$ & Debtor Expert 1 & Expert 2 & Expert 3 & Expert 4 & Expert 5 & Expert 6 \\
\hline Debtor's & B1 & 3 & 1 & 1 & 1 & 2 & 1 \\
financial & B2 & 4 & 4 & 2 & 2 & 4 & 3 \\
situation & B3 & 5 & 5 & 4 & 3 & 5 & 4 \\
Developme & B1 & 5 & 1 & 2 & 1 & 3 & 1 \\
nt prospect & B2 & 4 & 3 & 4 & 2 & 4 & 4 \\
of the loan & B3 & 1 & 4 & 5 & 4 & 5 & 5 \\
project & B3 & & & & & &
\end{tabular}

By marking the result data of financial situation of debtors in Table 1, have

$$
X_{1}=\left(\begin{array}{llllll}
3 & 1 & 1 & 1 & 2 & 1 \\
4 & 4 & 2 & 2 & 4 & 3 \\
5 & 5 & 4 & 3 & 5 & 4
\end{array}\right), F_{1}=X_{1}^{T} X_{1}=\left(\begin{array}{ccc}
17 & 31 & 53 \\
31 & 65 & 86 \\
53 & 86 & 116
\end{array}\right)
$$


By the exponential iterative calculation, have the eigenvector of the matrix $F_{1}$ 's corresponding biggest latent root

$$
x_{*_{1}}=\left(\begin{array}{lll}
0.3159, & 0.5589,0.7668
\end{array}\right)^{T}
$$

According to formula (3) and formula (4), we get the entropy values and weight values of each expert, which have been list in Table 2 , through evaluating the enterprises' financial situation.

Table 2. The entropy values and weight values of each expert ( $j=$ enterprises' financial situation)

\begin{tabular}{|c|c|c|c|c|c|}
\hline$S_{i}$ & \multicolumn{3}{|c|}{$E_{i}=\left(e_{i 1}, e_{i 2}, e_{i 3}\right)^{T}$} & $h_{i j}$ & $\omega_{i j}$ \\
\hline$S_{1}$ & $(0.8916$ & 0.9932 & $0.9403)^{T}$ & 0.1670 & 0.141 \\
\hline$S_{2}$ & $(0.8384$ & 0.9417 & $0.9953)^{T}$ & 0.2090 & 0.113 \\
\hline$S_{3}$ & $(0.9032$ & 0.8875 & $0.8288)^{T}$ & 0.3631 & 0.065 \\
\hline$S_{4}$ & $(0.9514$ & 0.9756 & $0.9650)^{T}$ & 0.1059 & 0.222 \\
\hline$S_{5}$ & $(0.9822$ & 0.9626 & $0.9786)^{T}$ & 0.0755 & 0.311 \\
\hline$S_{6}$ & $(0.8022$ & 0.9706 & $0.9823)^{T}$ & 0.1588 & 0.148 \\
\hline
\end{tabular}

Table 2 proves that expert3's cooperating is relatively bad because of his most heavy entropy value. Herewith, according the mark values of evaluating the loan project's development prospect, we could get the entropy values and weight values of each expert which have been list in Table 3 .

Table 3. The entropy values and weight values of each expert ( $j=$ loan project's development prospect)

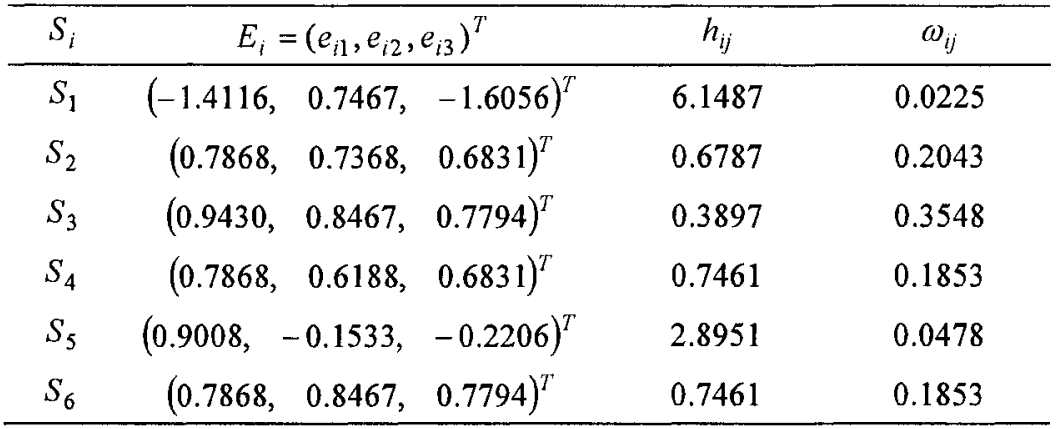

From table 3 , the entropy values of expert 1 and expert 5 are very much heavy, so their cooperation is very bad. We should consider replacing them. According to the formula (4), we receive the final result like Table 4. 
Table 4. Synthetically final result table

\begin{tabular}{cccc}
\hline Debtor's code $i$ & 1 & 2 & 3 \\
\hline Mark value $E_{i}$ & 3.1334 & 6.7031 & 8.8634 \\
\hline
\end{tabular}

The result is: $B_{3}>B_{2}>B_{1}$. So, the debtor ${ }^{B_{3}}$ is optimum.

\section{Conclusions}

Strengthening credit risk evaluation management to keep stability of the banking, it is significant to protect the healthy development of national economy. This paper quantitatively studies system cooperative model in the course of credit evaluation, effectively solves the problem of the many attributes of group decision, which is the credit evaluation system's key problem, prompts the banking to change the method of controlling and determining credit risk from qualitative observation while being past into quantitative analysis nowadays, improves accuracy and maneuverability of this kind of evaluation and decision. Certainly, it is very difficult to devote present theory research results into really practice use, so we should have much further research and discussion.

\section{Acknowledgements}

The Authors thank Professors Ye Xiang and Su Jun for sharing their insight and for their suggestions on the topic and research ideas of this paper.

This research was a foundation item of Project supported by the National Science Foundation of China (Grant No. 70501002) and National Ministry of Education of China: Research on IT systems management and evaluation model of performance, 2006 2007. 


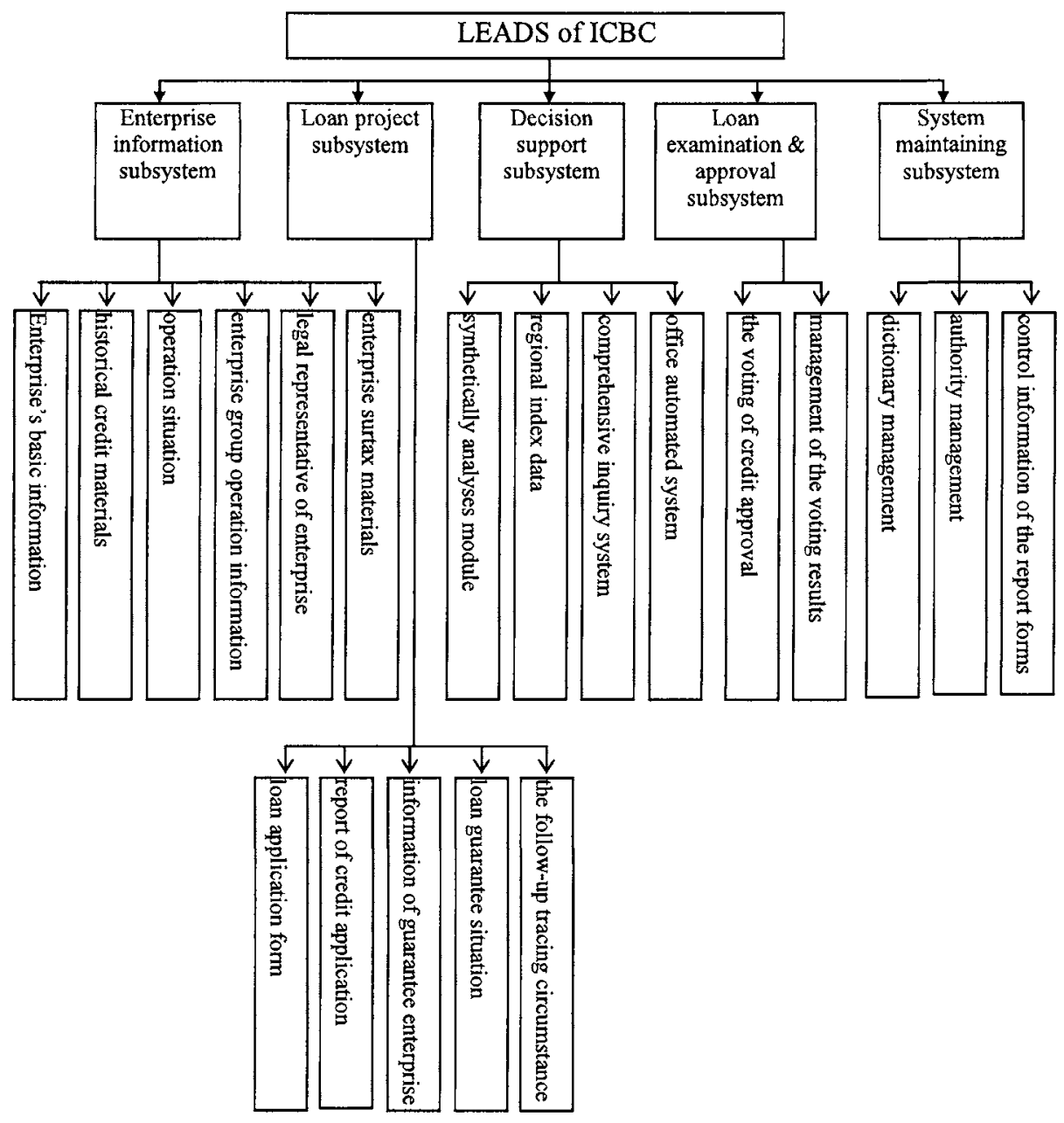

Fig. 2. The framework of LEADS

\section{References}

1. W.H Qiu, Decision and Application Entropy Studying (China Machine Press, Beijing, 2001) 2. W.H Qiu, The Entropy Model Of Group Decision, Control and Decision 10(1), 50-54, (1995).

3. W.H Qiu, The Latent Root Method Of Group Decision, Applied Mathematics and Mechanics 18(11), 1027-1031, (1997). 
4. H.X Jiang, Deposit Financial Institution's Credit Risks Theory Research And Its Application: PhD Diploma Paper, Beijing: BeiHang University, 2003.

5. Beijing Creming Science \& Technology Development Co. Ltd. Project Report of Loan Examination Decision Analysis System for ICBC, version 2.0 of Notes part, (unpublished) Beijing, 2000-8-18.

6. H.X Jiang, X. YE, IT Systems Management And Its Application In The Complicated Environment, In the Proceeding of 2005 International Conference on Construction and Real Estate Management, Penang, Malaysia, China Architecture \& Building Press, 2005, pp. 538 540. 